\title{
Vestibology
}

\section{Vestibular pathology and spatial working memory}

\author{
Patologia vestibolare e memoria operativa spaziale \\ Giorgio Guidetti, Riccardo Guidetti, Maurizio Manfredi, Marco Manfredi \\ Vertigo Centre, Poliambulatorio Chirurgico Modenese, Italy
}

\section{SUMMARY}

Thanks to wide central connections the vestibular system is not merely involved in reflexes, but it is also connected to cognitive processes. A growing body of literature suggests that it has a substantial impact on cognitive function. These cognitive interactions include memory, attention, mental imagery, body awareness and social cognition. Spatial working memory (SWM) is a kind of short-term memory that allows to temporarily store and manipulate spatial information. It has a limited capacity and is quite vulnerable to interference. The single most important nonverbal task for assessment of visuo-spatial working memory (VSWM) is the Corsi block tapping task (CBTT), also known as the Corsi Span Test. We evaluated 263 patients suffering from chronic unilateral or bilateral vestibular loss (VL) by eCorsi Block-Tapping test before and after 5 days of instrumental vestibular training (IVT). The data were compared with those of 834 subjects submitted to the same test: 430 healthy people (HP) and 404 patients suffering from chronic VL but not treated by IVT. At all ages, the Corsi block test score was extremely statistically significantly higher $(\mathrm{p}<0.0001)$ in HP than in both groups of VL. The score showed a statistically significant difference with age and sex in healthy subjects as younger males obtained the best results. Our study confirms the significant interference of the vestibular input on VSWM and impairment of this cognitive function in patients suffering from chronic UL or BIL. It also shows that IVT is able to improve VSWM even in cases where the deficit is greater.

KEY WORDS: spatial working memory, corsi block-tapping test, vestibular pathology, vestibular rehabilitation training

\section{RIASSUNTO}

Grazie ad ampie connessioni centrali il sistema vestibolare non evoca solo riflessi ma ̀̀ anche collegato a processi cognitivi. Un numero sempre maggiore di studi suggerisce che ha un impatto sostanziale sulle funzioni cognitive. Queste interazioni cognitive comprendono la memoria, l'attenzione, l'immaginario mentale, la consapevolezza del corpo e la cognizione sociale. La memoria operativa spaziale è un tipo di memoria a breve termine che consente di archiviare e manipolare temporaneamente le informazioni spaziali. Ha una capacità limitata ed è piuttosto vulnerabile alle interferenze. Il test non verbale più importante per la valutazione della memoria di lavoro visuo-spaziale (VSWM) è il test a blocchi di Corsi, noto anche come il Test di span di Corsi. A tal fine, abbiamo valutato col test eCorsi Block-Tapping 263 pazienti affetti da deficit vestibolare cronico unilaterale o bilaterale (VL), prima e dopo 5 giorni di training vestibolare strumentale (IVT). I dati sono stati confrontati con quelli di altri 834 soggetti sottoposti allo stesso test: 430 persone sane (HP) e 404 pazienti affetti da VL cronica ma non trattati con IVT. A tutte le età il punteggio del test di Corsi era significativamente più alto $(p<0,0001)$ negli HP rispetto a entrambi $i$ gruppi di VL. Il punteggio era significativamente più alto anche nei giovani rispetto ai vecchi sia nelle HP che nei VL. È significativamente più alto nei maschi che nelle femmine solo negli HP. Il nostro studio conferma l'interferenza significativa dell'input vestibolare sulla VSWM e la compromissione di questa funzione cognitiva in pazienti affetti da VL cronici mono o bilaterali. Esso mostra anche che IVT è in grado di migliorare la VSWM anche nei casi in cui il deficit è maggiore.

PAROLE CHIAVE: memoria operativa spaziale, test di corsi, patologia vestibolare, training riabilitativo vestibolare
Received: May 5, 2018

Accepted: October 6, 2018

Published on line: July 31, 2019

\section{Correspondence \\ Giorgio Guidetti \\ Vertigo Center - Poliambulatorio Chirurgico \\ Modenese, strada Morane 390, 41125 Modena, Italy \\ E-mail: giorgio.vertigine@gmail.com}

\section{Funding}

None.

\section{Conflict of interest}

The Authors declare no conflict of interest.

How to cite this article: Guidetti G, Guidetti R, Manfredi M, et al. Vestibular pathology and spatial working memory. Acta Otorhinolaryngol Ital 2020;40:72-78. https://doi.org/10.14639/0392$100 \mathrm{X}-2189$

๑ Società Italiana di Otorinolaringoiatria e Chirurgia Cervico-Facciale

\section{(c) (1) $\Theta($}

This is an open access article distributed in accordance with the CC-BY-NC-ND (Creative Commons Attribution-NonCommercial-NoDerivatives 4.0 International) license. The article can be used by giving appropriate credit and mentioning the license, but only for non-commercial purposes and only in the original version. For further information: https:// creativecommons.org/licenses/by-nc-nd/4.0/deed.en 


\section{Introduction}

The vestibular system consists of the peripheral vestibular organs in the inner ear and the associated extensive central nervous system projections to the cerebellum, brainstem, lymbic system, thalamus and cortex. The vestibular cortex differs in various ways from other sensory cortices. It consists of a network of several distinct and separate areas. Its core region, the parieto-insular vestibular cortex (PIVC), is located in the posterior insula and retroinsular region, and includes the parietal operculum. A ubiquitous aspect of central vestibular processing is its promiscuity given that vestibular signals are commonly found in combination with other sensory signals ${ }^{1}$. The entire network is multisensory (in particular, vestibular, visual and somatosensory) and bilaterally organised; there are various pontomesencephalic brainstem crossings and at least two transcallosal connections of both hemispheres, between the PIVC and the motion-sensitive visual cortex areas, which also mediate vestibular input. Bilateral organisation is a major key to understanding cortical functions and disorders, for example the visual-vestibular interaction that occurs in spatial orientation. Although the vestibular cortex is represented in both hemispheres, there is only one global percept of body position and motion ${ }^{2}$. This system is important for spatial orientation and balance, both of critical ecological importance, particularly for successful and safe navigation in our environment. However, little is still known regarding central vestibular processing and the brain regions that mediate vestibular motion, and vestibular spatial perception is an area of ongoing research ${ }^{3}$.

Thanks to these wide central connections, the vestibular system is not merely involved in reflexes but is also connected to cognitive processes. A growing body of literature suggests that it has a substantial impact on cognitive function. These cognitive interactions include memory, attention, mental imagery, body awareness and social cognition. Cognitive deficits such as poor concentration and shortterm memory loss are known by clinicians to occur frequently among patients with vestibular abnormalities of any type ${ }^{4,5}$, especially for the elderly ${ }^{6}$. For this reason, emerging research suggests the vestibular system can be considered as a potential window for exploring brain function beyond that of maintenance of balance, and into areas of cognitive, affective and psychiatric symptomology ${ }^{7}$.

Furthermore, the links between vestibular dysfunction and cognitive performance can suggest areas of future research and application ${ }^{7-9}$. Damage to the vestibular system specifically leads to cognitive deficits in spatial learning and memory, navigation, mental rotation and mental representation of three-dimensional space, which are not necessar- ily related to any particular episode of vertigo or dizziness, and therefore may occur even in patients who are otherwise well compensated.

Spatial memory is a cognitive process responsible for recording information about the spatial environment and spatial orientation. It enables a person to remember different locations as well as spatial relations between objects and allows one to remember where an object is in relation to another object. It can be short or long-term. Short-term memory (STM) allows to temporarily store and manage information that is necessary to complete complex cognitive tasks. Spatial working memory (SWM) is a kind of STM that allows to temporarily store and manipulate spatial information. It has a limited capacity and is quite vulnerable to interference. It is well known that the primate dorsolateral prefrontal cortex, especially the area surrounding the principal sulcus, participates in spatial working memory processes. These results indicate that the dorsolateral prefrontal cortex takes part in spatial working memory, especially in the temporary active storage of spatial information.

Recently, it was suggested that a network extending across several cortical areas, including the dorsolateral prefrontal cortex and posterior parietal cortex, takes part in spatial working memory. Human neuroimaging studies showed activation during the spatial working memory tasks in numerous cortical areas, including the dorsolateral prefrontal cortex, frontal eye field, supplementary motor area, premotor cortex, anterior cingulate cortex, posterior parietal cortex and occipital cortex. These results provide supporting evidence that the neuronal links between these cortical areas constitute part of a working memory system. In addition to closely intertwined attentional and oculomotor programming, SWM has also been shown to involve higher-order cognitive processes, such as executive functioning, at the earliest stages of information processing. Thus, depending on the strategies elaborated and task demands, the same spatial information may be represented in SWM by different patterns of activation in the brain. This view is consistent with a model of WM arising from the interaction between higher-order cognitive top down processes governed by the prefrontal cortex and stimulus specific brain regions. Visual attention has a cross-modal influence on activity in the human vestibular cortex: higher attentional loads were associated with decreasing activation in the anterior part of the PIC, whereas no such effect was observed for the posterior PIC. Brain activation was larger for visual attention (VA) tasks than for working memory WM) tasks, but deactivation was larger for WM tasks. VA and WM tasks commonly deactivate a network that includes the frontal, temporal, occipital, and limbic lobes; b) although WM 
tasks caused lower overall activation, they produced larger overall deactivation than VA tasks; and c) specific regions in the frontal lobes (PreCG, and PCL) deactivated during $\mathrm{WM}$, but activated during VA tasks ${ }^{10-13}$.

Dorsolateral prefrontal cortex (DLPFC) has been shown to be a part of the SWM network, but its specific functional role remains unknown ${ }^{14}$.

It has been speculated that stimulating the vestibular system during balance training may induce changes of the hippocampus and parietal cortex possibly via direct pathways between the vestibular system and these brain regions ${ }^{7-9,15-20}$ and chronically dizzy patients seem to profit from combining training with medication or brain stimulation.

Neither the side of the vestibular lesion nor the duration of disease seem to influence cognitive performance, but the degree of vestibular dysfunction significantly correlates with cognitive deficit.

The single most important nonverbal task for the assessment of visuo-spatial working memory (VSWM) is the Corsi block tapping task (CBTT), also known as the Corsi Span Test ${ }^{21}$. It also involves spatial attention. The traditional version of Corsi apparatus consists of a set of nine identical blocks $(3 \times 3 \times 3 \mathrm{~cm})$ irregularly positioned on a wooden board $(23 \times 28 \mathrm{~cm})$. The experimenter points to a series of blocks at a rate of one block per second. Subsequently, the participant is required to point to the same blocks in their order of presentation. The length of the block sequences (starting from 2-block sequences) increases by one item until recall is no longer correct. The procedure ends when the number of wrong reproductions exceeds the proportion of admissible errors per length. A span score is calculated corresponding to the larger sequence the subject can correctly reproduce. The maximum score possible is 9 . Some changes have been suggested over time ${ }^{22}$. Several computer-based forms of the test have been developed ${ }^{23}$ or by using a mouse to click on the blocks, as well as more peculiar versions of CBTT such as a haptic Corsi and a "walking Corsi".

In Corsi block-tapping task for digital tablets (eCorsi), instead of cubes to be tapped on a board, the setup consists of squares that flash on a computer screen. Participants reproduce the sequences either by tapping blocks on a (touch) screen without substantial differences between the two versions in terms of subjects' performance ${ }^{24}$. On average, most participants achieve a span of five items on the Corsi span test and seven on the digit span task. Differences between traditional CBTT and eCorsi include mostly better control of the inter-stimulus presentation timings in the eCorsi. In fact, with manual tapping the temporal accuracy is particularly difficult to control by the examiner, who can (inadvertently) be slower or faster depending on several factors.
This test is used mainly in neurological diseases (Alzheimer's disease. autism spectrum disorder, depression and affective disorders, Down's syndrome, epilepsy, multiple sclerosis, Parkinson's disease, schizophrenia, stroke and cerebrovascular disease and traumatic brain injury).

Spatial working memory problems are frequently reported following brain damage within both left and right hemispheres, but with the severity often being greater in individuals with right hemisphere lesions. Patients with damage to the right posterior parietal cortex, the dorsolateral prefrontal cortex and hippocampal formation bilaterally made more between-search errors, indicating the importance of these areas in maintaining spatial information in working memory over an extended period of time.

At the Corsi Block Tapping Test, both bilateral and unilateral vestibular patients are significantly impaired in their visuospatial abilities compared with healthy controls ${ }^{4,24}$.

SWM declines across the life span even in the absence of disease-related cerebral pathology ${ }^{25}$. One reason for this decline is certainly working memory-related functional cerebral changes, particularly within the prefrontal cortex ${ }^{26}$. Mean span capacity increased incrementally and linearly with age, and no gender difference was observed. The increase in performance with advancing age supports the notion that spatial immediate memory capacity increases with maturation throughout childhood. Comparisons indicated that the span capacity of eighth graders is not statistically different from that of young adults, suggesting an upper developmental plateau for spatial span in early adolescence with an ameliorative effect of education. Taskrelated functional connectivity appears to be lower in older adults with age-related reductions of prefrontal activation during spatial working memory retrieval. Performance accuracy in older adults is associated with right dorsolateral and anterior prefrontal cortex activation, and with the functional connection between these regions ${ }^{26}$. Sex differences are often reported in spatial abilities. Until a few years ago, it was widely accepted that men outperformed women on almost all spatial tasks. However, some studies show conflicting results, which can be ascribed to the complexity of the variables involved in the visuo-spatial domain and can be better explained by differences in spatial competences. Indeed, these differences could reflect the use of different strategies, rather than different competences, by the two sexes.

\section{Materials and methods}

There is moderate to strong evidence that vestibular rehabilitation is a safe, effective management for unilateral peripheral vestibular dysfunction, based on a number of 
high-quality randomised controlled trials ${ }^{27}$ even in older people ${ }^{28}$. The goal of the present study was to test the hypothesis that a full-immersion vestibular instrumental training is able to improve SWM in patients suffering from chronic vestibular loss, defined by the presence of reduced function of the peripheral vestibular system on one side, which has persisted for 3 or more months. In humans, balance skills have been associated ${ }^{29}$ with an increased volume of the hippocampus, basal ganglia and frontal and parietal brain areas. However, data on the effects of balance training on cognitive functions, particularly related to memory and spatial cognition, are rare to date and limited to healthy people ${ }^{30}$. To this end, we evaluated patients suffering from vestibular loss (VL) by eCorsi Block-Tapping test before and after 5 days of instrumental vestibular training (IVT).

We examined 263 subjects with chronic vestibular loss (TVL):

- 154 (58.6\%) females (F) and 109 (41.4\%) males (M);

- 150 cases (YOUNG) aged 17 to 64 years (mean 48.3 SD 10.7);

- 113 cases (OLD) aged 65 years or more (mean 75.0 SD 5.21).

VL was unilateral right (RL) in 98 cases, unilateral left (LL) in 67 cases and bilateral (BL) in 98 cases.

Subjects on therapy with psychoactive or antiepileptic drugs, suffering from degenerative, neoplastic or severe vascular pathologies of the central nervous system, Meniere's disease, or paroxysmal positional vertigo were not included in the study. Patients with small age-related vascular signs in imaging tests were included.

The vestibular training program consisted of 8 sessions of one hour in 5 consecutive days. Both static and dynamic platforms of various kinds and treadmills were used. Optokinetic stimulations and visual targets of various types were associated with static and dynamic exercises. The degree of stimulation was adapted to the basic performance of each patient and was progressively increased day by day.

Our version of the eCorsi apparatus consisted of a set of nine identical squares of $3 \mathrm{~cm}$ irregularly positioned that flash on a touch screen monitor at a rate of one block per second.

Participants must repeat the correct sequence by tapping blocks on the screen in the order of appearance. The length of the block sequences (starting from a 2-block sequence) increases by one item until the subject fails to recall the correct sequence. A span score, that corresponds to the larger sequence the subject is able to correctly reproduce, was calculated. The maximum score was 9 .

The data were compared with those of other 834 subjects submitted to the same test:
- 430 healthy people (HP); 224 F (52.1\%) and $206 \mathrm{M}$ (47.9\%):

- 300 aged 17 to 64 years (mean 31.57 SD 12.41);

- 130 aged 65 years or more (mean 72.26 SD 4.79);

- 404 patients suffering from chronic vestibular loss, but not treated by vestibular training (NTVL): $210 \mathrm{~F}$ (51.9) and $194 \mathrm{M}(48.1 \%)$ :

- 234 aged 17 to 64 years (mean 48,94 SD 10.44);

- 170 aged 65 years or more (mean 75.08 SD 5.25).

Statistical analysis was performed using SPSS (Statistical Package for Social Science). Groups were tested for normality. The difference between the means of normally distributed variables was calculated using Student's t-test. A paired sample t-test was performed to assess the differences in the same subject, while independent samples ttest was used to compare two groups. Differences with a p-value $<0.05$ were considered statistically significant.

\section{Results}

For all age groups the Corsi block test score reached a high level of statistical significance $(\mathrm{p}<0.0001)$ between HP and NTVL and TVL ( $<$ 0.0001) (Tabs. I, II).

In the HP group, the span was significantly higher in M than in F (Tab. III). This difference was not significant in the VL and NTVL groups, while it reached significance in TVL (p 0.0068) (Tabs. III, IV).

In both HP and NTVL the score was significantly higher in YOUNG than in OLD (Tabs. III, IV) $(\mathrm{p}<0.0001$ and $\mathrm{p}<0.0001$ respectively).

The difference between UL and BIL remained unclear. In fact, in NTVL was not significant, while it was slightly sig-

Table I. Mean and SD values of the span of eCorsi in HP and NTVL.

\begin{tabular}{lcccccc} 
& \multicolumn{3}{c}{ HP } & & \multicolumn{3}{c}{ NTVL } \\
& N & Mean & SD & N & Mean & SD \\
M & 206 & 6.374 & 0.922 & 194 & 4.804 & 1.226 \\
F & 224 & 6.004 & 1.022 & 210 & 4.666 & 3.587 \\
YOUNG & 300 & 6.58 & 0.778 & 234 & 5.184 & 3.387 \\
OLD & 130 & 5.294 & 0.774 & 170 & 4.112 & 1.074 \\
YOUNG M & 142 & 6.781 & 0.685 & 108 & 5.25 & 1.103 \\
OLD M & 64 & 5.468 & 0.712 & 85 & 4.23 & 1.138 \\
YOUNG F & 158 & 6.399 & 0.813 & 126 & 5.12 & 4.527 \\
OLD F & 66 & 5.090 & 0.700 & 85 & 4.00 & 1.000 \\
RL & & & & 160 & 5.237 & 4.029 \\
LL & & & & 118 & 4.432 & 1.343 \\
BL & & & & 126 & 4.373 & 0.986 \\
UL & & & & 278 & 4.896 & 3.200 \\
Total & 430 & 6.181 & 0.991 & 404 & 4.732 & 2.720 \\
\hline
\end{tabular}


Table II. Mean and SD values of the span of eCorsi in TVL.

\begin{tabular}{lccccc} 
& \multicolumn{2}{c}{ Before IVT } & \multicolumn{2}{c}{ After IVT } \\
& N & Mean & SD & Mean & SD \\
M & 109 & 5.119 & 1.420 & 5.598 & 1.326 \\
F & 154 & 4.664 & 1.267 & 4.914 & 1.266 \\
YOUNG & 150 & 5.267 & 1.251 & 5.607 & 1.236 \\
OLD & 113 & 4.310 & 1.150 & 4.646 & 1,172 \\
YOUNG M & 58 & 5.119 & 1.421 & 5.598 & 1.326 \\
OLD M & 51 & 4.285 & 1.214 & 4.735 & 1.024 \\
YOUNG F & 92 & 5.043 & 1.176 & 5.293 & 1.143 \\
OLD F & 62 & 3.937 & 1.119 & 4.187 & 1.179 \\
RL & 98 & 5.173 & 1.227 & 5.418 & 1.201 \\
LL & 67 & 4.701 & 1.360 & 5.149 & 1.329 \\
BL & 98 & 4.643 & 1.270 & 5.0 & 1.377 \\
UL & 165 & 4.988 & 1.300 & 5.309 & 1.257 \\
Total & 263 & 4.855 & 1.297 & 5.193 & 1.297 \\
\hline
\end{tabular}

Table III. P value in t-test between HP and NTVL groups and between NTVL subgroups by age, gender and kind of $\mathrm{VL}$.

\begin{tabular}{lc} 
Groups & P value in t-test \\
HP/NTVL & $<0.0001$ \\
M HP/M NTVL & $<0.0001$ \\
M/F HP & $<0.0001$ \\
M/F NTVL & 0.6110 not significant \\
F HP/F NTVL & $<0.0001$ \\
YOUNG/OLD HP & $<0.0001$ \\
YOUNG HP/YOUNG NTVL & $<0.0001$ \\
OLD HP/OLD NTVL & $<0.0001$ \\
YOUNG/OLD NTVL & $<0.0001$ \\
UI/BIL NTVL & 0.0734 not significant \\
RL/LL NTVL & 0.0379 \\
\hline
\end{tabular}

nificant in TVL $(p=0.0368)$, with a lower span in BIL (Tabs. III, IV)

Both in NTVL and in TVL the span was significantly higher in RL than in LL (Tab. IV). The difference between HP and TVL remained significant even after IVT.

However, IVT was able to significantly improve the span (Tab. IV). In fact, before IVT there was no statistically significant difference between NTVL and TVL, while there was a marked improvement in TVL after IVT $(p=0.0029)$ (Tab. IV). On average this improvement was significant in M ( $p=0.0107)$ (Tab. IV). The difference between YOUNG and OLD remained significant, but the improvement of the score was significant in both groups, with a high level of significance in OLD F $(\mathrm{p}<0.0001)$. UL improved significantly more than BIL, without significant differences be- tween RL and LL. However, the difference between UL and BIL was not statistically significant.

\section{Discussion}

With a greater number of cases than previously studied, our study confirmed a very significant VSWM deficit in patients suffering from unilateral or bilateral VL at all ages. It is therefore clear that vestibular input is able to interact in a relevant, chronic way even with this cognitive function. The deficit is greater in the OLD, both in HP and in $\mathrm{VL}$, compatibly with the condition of cognitive decay due to age.

The partially worse VSWM deficit in the LL compared to the RL could find explanation in the different ways of projection of the vestibular input on the two sides of the cerebral cortex. Vestibular cortical activations after unilateral caloric ear irrigation was measured depending on both the stimulated ear and the handedness, with a predominant activation of the ipsilateral vestibular cortex following right

Table IV. $P$ value in t-test between HP, NTVL and TVL groups and between TVL subgroups by age, gender and kind of VL before and after IVT.

\begin{tabular}{lc} 
Groups before IVT & P value in t-test \\
HP/TVL & $<0.0001$ \\
NTVL/TVL & 0.4940 not significant \\
M/F & 0.0068 \\
YOUNG/OLD & $<0.0001$ \\
UL/BIL & 0.0368 \\
RL/LL & 0.0215 \\
Groups after IVT & P value in t-test \\
HP/TVL & $<0.0001$ \\
NTVL/TVL & 0.0105 \\
TVL after/TVL before & 0.0029 \\
M/F & $<0.0001$ \\
M after/M before & 0.0107 \\
F after/F before & 0.0843 not significant \\
YOUNG /OLD & $<0.0001$ \\
YOUNG after/YOUNG before & 0.0185 \\
OLD after/OLD before & 0.0307 \\
YOUNG M after/YOUNG M before & 0.0631 not significant \\
OLD M after/ OLD M before & 0.0457 \\
YOUNG F after/YOUNG F before & 0.1452 not significant \\
OLD F after/OLD F before & $<0.0001$ \\
UL after/UL before & 0.0232 \\
RL after/RL before & 0.1594 not significant \\
LL after/LL before & 0.0559 not significant \\
BIL after/BIL before & 0.0607 not significant \\
UL/BIL & 0.0641 not significant \\
\hline & \\
\hline &
\end{tabular}


or left caloric ear irrigation. These patterns of vestibular projections in humans, suggesting an importance of the ipsilateral pathways, and thus do not fully correspond with animal data that have stressed bilateral pathways with a contralateral dominance. In general, multiple studies have, however, demonstrated the dominance of the right hemisphere after vestibular stimulation. The activation in the right hemisphere is the same for both right- and left-ear stimulation. At the present time, it seems to us that this hypothesis is not sufficiently demonstrated with regard to VSWM.

The level of high statistical significance of the difference of HP between genders, with lower span in F compared to $\mathrm{M}$, is not as clear in the case of VL. We therefore think that in M the VL involves a greater deficit in the VSWM and therefore reduces the difference between genders. As far as navigation is concerned, various studies have shown that the gender difference or strategies depends on neural substrates. $\mathrm{M}$ uses more vestibular inputs than $\mathrm{F}$, which has a strategy more related to environmental visual inputs ${ }^{27}$. Indeed, $\mathrm{F}$ typically report navigating on the basis of local landmarks and familiar routes, whereas $\mathrm{M}$ report using cardinal directions, environmental geometry and metric distances. Although F do not differ from $\mathrm{M}$ in dependence on or ability to use landmarks, they depend less on geometry when reorienting to an environment and are relatively more impaired at finding a target based on directional cues. $\mathrm{F}$ also require more environmental cues to remain oriented in an environment and have difficulty following navigation directions based on cardinal directions and metric distances. However, there are no other studies able to demonstrate that this is valid even for VSWM.

The IVT has been shown able to significantly improve VSWM, especially in cases with greater initial deficit, such as OLD F. It may be speculated that the vestibular system during balance training may induce changes of the hippocampus and parietal cortex possibly via direct pathways between the vestibular system and these brain regions ${ }^{15,16}$. It can, therefore, be assumed that the vestibular and visuovestibular stimulations during the 5 days of IVT is able to activate those neuronal circuits that in the case of VL do not guarantee a normal VSWM. It will be necessary to demonstrate in the future if longer training will allow to completely eliminate the difference between VL and HP.

\section{Conclusions}

Our study confirms the significant interference of the vestibular input on VSWM and the impairment of this cognitive function in patients suffering from chronic UL or BIL. It also showed that IVT is able to improve VSWM even in cases in which the deficit is greater. This result seems possible even without specific exercises for VSWM. Thus, new frontiers can be opened in the evaluation and treatment of chronic VL and in the study of neuronal circuits related to cortical projections of vestibular inputs.

\section{References}

1 Guidetti G. The role of cognitive processes in vestibular disorders. Hearing Balance Commun 2013;11(Suppl. 1):3-35. https://doi.org/10 $.3109 / 21695717.2013 .765085$

2 Dieterich M, Brandt T. The parietal lobe and the vestibular system. Handb Clin Neurol 2018;151:119-40. https://doi.org/10.1016/B9780-444-63622-5.00006-1

3 Seemungal BM. The components of vestibular cognition-motion versus spatial perception. Multisens Res 2015;28:507-24.

4 Guidetti G, Monzani D, Trebbi M, et al. Impaired navigation skills in patients with psychological distress and chronic peripheral vestibular hypofunction without vertigo. Acta Otorhinolaryngol Ital 2008;28:21-5.

5 Lopez C. The vestibular system: balancing more than just the body. Curr Opin Neurol 2016;29:74-83. https://doi.org/10.1097/ WCO.0000000000000286

6 Smith PF. The vestibular system and cognition. Curr Opin Neurol 2017;30:84-9. https://doi.org/10.1097/WCO.0000000000000403

7 Bigelow RT, Agrawal Y. Vestibular involvement in cognition: visuospatial ability, attention, executive function, and memory. J Vestib Res 2015;25:73-89. https://doi.org/10.3233/VES-150544

8 Medendorp WP, Selen LJP. Vestibular contributions to high level sensorimotor functions. Neuropsychologia 2017;105:144-52. https://doi. org/10.1016/j.neuropsychologia.2017.02.004

9 Besnard S, Lopez C, Brandt T, et al. Editorial: the vestibular system in cognitive and memory processes in mammalians. Front Integr Neurosci 2015;9:55. https://doi.org/10.3389/fnint.2015.00055

10 Gurvich C, Maller JJ, Lithgow B, et al. Vestibular insights into cognition and psychiatry. Brain Res 2013;1537:244-59. https://doi. org/10.1016/j.brainres.2013.08.058

11 Dieterich M, Brandt T. The bilateral central vestibular system: its pathways, functions, and disorders. Ann N Y Acad Sci 2015;1343:1026. https://doi.org/10.1111/nyas.12585

12 Mast FW, Preuss N, Hartmann M, et al. Spatial cognition, body representation and affective processes: the role of vestibular information beyond ocular reflexes and control of posture. Front Integr Neurosci 2014;8:44. https://doi.org/10.3389/fnint.2014.00044

13 zu Eulenburg P, Caspers S, Roski C, et al. Meta-analytical definition and functional connectivity of the human vestibular cortex. Neuroimage 2012;60:162-9. https://doi.org/10.1016/j.neuroimage.2011.12.032

14 Wu YJ, Tseng P, Chang CF, et al. Modulating the interference effect on spatial working memory by applying transcranial direct current stimulation over the right dorsolateral prefrontal cortex. Brain Cogn 2014;91:87-94. https://doi.org/10.1016/j.bandc.2014.09.002

15 Rogge AK, Röder B, Zech A, et al. Balance training improves memory and spatial cognition in healthy adults. Sci Rep 2017;7:5661. https://doi.org/10.1038/s41598-017-06071-9

16 Hötting K, Röder B. Beneficial effects of physical exercise on neuroplasticity and cognition. Neurosci Biobehav Rev 2013;37:2243-57. https://doi.org/10.1016/j.neubiorev.2013.04.005

17 Hitier M, Besnard S, Smith PF. Vestibular pathways involved in cognition. Front Integr Neurosci 2014;8:59. https://doi.org/10.3389/fnint.2014.00059 
18 Dilda V, MacDougall HG, Curthoys IS, et al. Effects of galvanic vestibular stimulation on cognitive function. Exp Brain Res 2012;216:275-85. https://doi.org/10.1007/s00221-011-2929-Z

19 Smith PF, Darlington CL, Zheng Y. Move it or lose it - is stimulation of the vestibular system necessary for normal spatial memory? Hippocampus 2010;20:36-43. https://doi.org/10.1002/hipo.20588

20 Popp P, Wulff M, Finke K, et al. Cognitive deficits in patients with a chronic vestibular failure. J Neurol 2017;264:554-63. https://doi. org/10.1007/s00415-016-8386-7

21 Richardson JT. Measures of short-term memory: a historical review. Cortex 2007;43:635-50.

22 Monaco M, Costa A, Caltagirone C, et al. Forward and backward span for verbal and visuo-spatial data: standardization and normative data from an Italian adult population. Neurol Sci 2013;34:749-54. https:// doi.org/10.1007/s10072-012-1130-x

23 Brunetti R, Del Gatto C, Delogu F. eCorsi: implementation and testing of the Corsi block-tapping task for digital tablets. Front Psychol 2014;5:939. https://doi.org/10.3389/fpsyg.2014.00939

24 Popp P, Wulff M, Finke K, et al. Cognitive deficits in patients with a chronic vestibular failure. J Neurol 2017;264:554-63. https://doi. org/10.1007/s00415-016-8386-7

25 Holtzer R, Rakitin BC, Steffener J, et al. Age effects on load- dependent brain activations in working memory for novel material. Brain Res 2009;1249:148-61. https://doi.org/10.1016/j.brainres.2008.10.009

26 Toepper M, Markowitsch HJ, Gebhardt H, et al. The impact of age on prefrontal cortex integrity during spatial working memory retrieval. Neuropsychologia 2014;59:157-68. https://doi.org/10.1016/j.neuropsychologia 2014.04.020

27 McDonnell MN, Hillier SL. Vestibular rehabilitation for unilateral peripheral vestibular dysfunction. Cochrane Database Syst Rev 2015;1:CD005397. https://doi.org/10.1002/14651858.CD005397. pub4

28 Howe TE, Rochester L, Neil F, et al. Exercise for improving balance in older people. Cochrane Database Syst Rev 2011;(11):CD004963. https://doi.org/10.1002/14651858.CD004963.pub3

29 Taubert M, Draganski B, Anwander A, et al. Dynamic properties of human brain structure: learning-related changes in cortical areas and associated fiber connections. J Neurosci 2010;30:11670-7. https://doi. org/10.1523/JNEUROSCI.2567-10.2010

30 Dordevic M, Hokelmann A, Muller P, et al. Improvements in orientation and balancing abilities in response to one month of intensive slackline-training. A randomized controlled feasibility study. Front Hum Neurosci 2017;11:55. https://doi.org/10.3389/fnhum.2017.00055 